\title{
Dor, ansiedade e qualidade de vida relacionada à saúde bucal de pacientes atendidos no serviço de urgência odontológica
}

\author{
Pain, anxiety and quality of life related to the oral health \\ of patients treated in a dental emergency clinic
}

\begin{abstract}
Mariane Flauzino Queiroz (https://orcid.org/0000-0001-6806-6805) ${ }^{1}$ Flaviana Dornela Verli (https://orcid.org/0000-0003-4241-4319) ${ }^{1}$ Sandra Aparecida Marinho (https://orcid.org/0000-0002-5379-8779) ${ }^{1}$ Paula Cristina Pelli Paiva (https://orcid.org/0000-0002-5960-4760) ${ }^{1}$ Suelleng Maria Cunha Santos (https://orcid.org/0000-0002-0452-3744) ${ }^{1}$ Janir Alves Soares (https://orcid.org/0000-0002-6100-4094) ${ }^{1}$
\end{abstract}

${ }^{1}$ Departamento de Odontologia, Faculdades de Ciências Biológicas e da Saúde, Universidade Federal dos Vales do Jequitinhonha e Mucuri. R. Glória 187, Centro. 39100-000 Diamantina MG Brasil. marianeflauzino@ hotmail.com

\begin{abstract}
The scope of this study was to assess the association between preoperative pain, anxiety and the impact of the oral condition on the quality of life of patients treated at the Dental Emergency Service at the Federal University of the Jequitinhonha and Mucuri Valleys. A retrospective cross-sectional study was conducted evaluating data from 240 patient charts aged from 18 to 65 years. Pain was measured by the Heft-Parker Visual Analog Scale; dental anxiety by Corah's Dental Anxiety Scale; and oral health-related quality of life by the Oral Health Impact Profile (OHIP-14). Associations were analyzed using Pearson's Chi-square test $(p<0.05)$. A statistically significant association was observed between pain and poorer quality of life, with impacts on psychological discomfort $(p<0.001)$, physical incapacity $(p<0.001)$ and the psychological $(p<$ $0.001)$ and social $(p<0.001)$ domains. Anxiety influenced poorer quality of life, with impacts on the psychological discomfort $(p=0.009)$, physical disability $(p=0.016)$, psychological disability $(p=0.011)$ and social disadvantage $(p=0.028)$ domains. This study is relevant for the planning of programs and actions aimed at the oral health of patients treated in dental emergency services, prioritizing those with the greatest psychosocial impact arising from oral problems.
\end{abstract}

Key words Pain, Dental treatment anxiety, Dental care, Quality of life
Resumo $O$ objetivo deste estudo foi avaliar associação entre dor pré-operatória, ansiedade $e$ impacto da condição bucal na qualidade de vida dos pacientes atendidos no serviço de Urgência Odontológica da Universidade Federal dos Vales do Jequitinhonha e Mucuri. Estudo transversal retrospectivo foi conduzido avaliando dados clínicos de 240 prontuários de pacientes com idades entre 18 e 65 anos. A dor foi mensurada pela Heft-Parker Visual Analogue Scale; ansiedade, pela Corah's Detal Anxiety Scale e qualidade de vida, pelo Oral Health Impact Profile (OHIP-14). Associações foram analisadas pelo Teste qui-quadrado de Pearson $(p<0,05)$. Foi observada associação estatisticamente significante entre dor e pior qualidade de vida, com impacto nos domínios desconforto psicológico $(p<0,001)$, incapacidade física $(p<0,001)$, psicológica $(p<0,001)$ e social $(p<$ 0,001 ). Ansiedade influenciou na pior qualidade de vida, com impacto nos dominios desconforto psicológico $(p=0,009)$, incapacidade fisica ( $p$ $=0,016)$, psicológica $(p=0,011)$ e desvantagem social $(p=0,028)$. Esse estudo é relevante para o planejamento de ações voltadas para a saúde bucal dos pacientes atendidos nos serviços de urgência odontológica, priorizando aqueles com maiores impactos psicossociais decorrentes dos problemas bucais.

Palavras-chave Dor, Ansiedade ao tratamento odontológico, Assistência odontológica, Qualidade de vida 


\section{Introdução}

A urgência odontológica (UO) consiste em medidas imediatas que visam a aliviar os sintomas dolorosos, infecciosos e estéticos da cavidade bu$\mathrm{cal}^{1}$. No atendimento de UO, tanto em consultórios como em escolas de odontologia, a procura do paciente é motivada, na maioria das vezes, por uma queixa de dor ${ }^{2-6}$. A prevalência de dor de dente reportada em estudos nacionais e internacionais variou de 7 a $36 \%$, em pacientes acima de 16 anos $^{2-6}$.

Devido à sintomatologia dolorosa, os problemas de saúde bucal podem restringir as funções cotidianas, culminando em afastamento das atividades escolares ou trabalhistas, provocando sofrimento e impactando negativamente na qualidade de vida ${ }^{7-11}$. Além da dor, deficiências estéticas causadas pela cárie dentária, fraturas ou hábitos parafuncionais são também motivos de procura por atendimento na UO. Estas alterações podem determinar diminuição da autoconfiança pessoal, repercutindo negativamente nas relações sociais, econômicas, educacionais e/ou ocupacionais ${ }^{7-11}$.

Com o intuito de compreender os efeitos provocados por problemas bucais na vida diária das pessoas, foi desenvolvido o Oral Health Impact Profile (OHIP) $)^{12}$, sendo uma das medidas de avaliação de saúde bucal e qualidade de vida mais sofisticadas e amplamente utilizada, apresentando implicações importantes para a prática clínica e na pesquisa odontológica ${ }^{13-15}$. O questionário validado foi utilizado em vários ramos da odontologia para avaliar o impacto de diferentes métodos terapêuticos sobre a qualidade de vida relacionada à saúde bucal do paciente, tanto em estudos transversais quanto longitudinais ${ }^{13-15}$.

A ansiedade ao tratamento consiste em um sentimento alimentado por situações relacionadas ao atendimento odontológico, que causam apreensão e desconforto, o que culmina, muitas vezes, em barreira na procura do atendimento, agravando a situação bucal, gerando dor e desconforto, criando assim uma expectativa negativa no paciente ${ }^{16}$. Estudos reportaram que a ansiedade ao tratamento odontológico pode impactar na qualidade de vida ${ }^{2,17,18}$.

Apesar do reconhecimento da importância dos aspectos sociais, econômicos e psicológicos na determinação da doença, a odontologia continua empregando, quase que exclusivamente, índices biológicos na avaliação e determinação das necessidades de tratamento e apreciação de programas de saúde bucal ${ }^{19}$. Uma das limitações desses índices biológicos é o fato de não consi- derarem a percepção subjetiva do indivíduo em relação à saúde bucal e não avaliarem a maneira como a saúde bucal afeta sua vida diária ${ }^{20}$. O uso de indicadores sociodentais, baseados na autopercepção bem como na avaliação da ansiedade em relação ao tratamento odontológico e o impacto destes indicadores na qualidade de vida dos indivíduos, oferece vantagens importantes para o planejamento e a provisão dos serviços odontológicos, com a mudança da ênfase de aspectos puramente biológicos para aspectos psicológicos e sociais ${ }^{19-21}$.

Íntima relação pode ser sugerida entre ansiedade ao tratamento odontológico e dor, em que pacientes evitam as visitas rotineiras, procurando apenas os atendimentos de UO, principalmente para alívio da sintomatologia dolorosa. Portanto, o presente estudo objetivou avaliar a associação existente entre a dor pré-operatória, a ansiedade e o impacto da condição bucal na qualidade de vida dos pacientes atendidos no setor de urgência odontológica. A hipótese testada foi que pacientes com sintomatologia dolorosa são mais ansiosos e que a condição bucal impacta negativamente na qualidade de vida.

\section{Metodologia}

\section{Desenho do estudo}

Estudo transversal com análise dos registros dos prontuários de pacientes adultos atendidos no Setor de Urgência Odontológica (SUO) da Universidade Federal dos Vales do Jequitinhonha e Mucuri (UFVJM).

\section{Amostra}

A população do estudo incluiu prontuários odontológicos de pacientes com idade superior a 18 anos de ambos os sexos, sem discriminaçao de cor e condição socioeconômica. Foram excluídos os prontuários de pacientes que não foram preenchidos corretamente ou com ausência dos dados.

As idades dos pacientes foram categorizadas pela mediana em: 18 e 35 anos e acima de 36 anos de idade.

\section{Coleta de dados}

A coleta de dados foi realizada por um único pesquisador, previamente treinado, que analisou os prontuários do SUO da UFVJM correspondentes ao período de um ano de atendimento, de 
2014 a 2015. O treinamento e a calibração foram realizados seguindo os preceitos propostos pela Organização Mundial da Saúde (1993). Primeiro foi realizada orientação teórica sobre os itens constante na ficha clínica epidemiológica e nos questionários incluindo os índices, os códigos e os critérios utilizados, momento em que foi esclarecido o maior número possível de dúvidas relativas aos critérios. Posteriormente, foram realizados o treinamento e a calibração prática, quando o pesquisador analisou os prontuários dos pacientes atendidos no SUO e inseriu os resultados no banco de dados, simultaneamente com o orientador. Este procedimento foi reproduzido uma semana depois para avaliar a concordância inter e intra-examinador, obtendo-se os valores de Kappa de 0,89 e 0,95.

\section{Instrumentos para coleta de dados}

Os prontuários continham três instrumentos, que foram aplicados antes do atendimento odontológico de cada paciente: (1) Avaliação da dor pré-operatória, segundo a escala Heft-Parker Visual Analogue Scale (HP-VAS); (2) Avaliação da ansiedade pré-operatória, segundo a escala de ansiedade odontológica Corah's Detal Anxiety Scale (CDAS) e (3) Oral Health Impact Profile (OHIP-14). A aplicação destes instrumentos faz parte do protocolo de atendimento do SUO da UFVJM, sendo realizada pelos alunos do $7^{\circ}$ e $8^{\circ}$ períodos do curso de odontologia, que são previamente calibrados e supervisionados pelos docentes responsáveis pelo SUO.

O HP-VAS consiste em uma reta com marcações milimetricamente definidas que correspondem a termos que descrevem níveis de dor: sem, fraca, suave, moderada, forte e intensa. Assim, existe uma equivalência entre a intensidade da dor e a posição marcada pelo paciente na reta, indicando subjetivamente a dor atual sentida antes do atendimento (dor pré-operatória). Posteriormente, o operador mede o local correspondente à marcação do paciente e faz a classificação baseada nas categorias propostas ${ }^{22}$.

A CDAS foi elaborada por Corah em $1969^{23} \mathrm{e}$ validada no Brasil ${ }^{24}$. O instrumento é composto de quatro perguntas: "(1) Se você tivesse que ir ao dentista amanhã como se sentiria?; (2) Quando se encontra na sala de espera do ambulatório, esperando ser chamado pelo dentista, como se sente?; (3) Quando você se encontra na cadeira do dentista aguardando que ele inicie os procedimentos de anestesia local, como se sente?; (4)Você está na cadeira do dentista já anestesiado. Enquanto aguarda o dentista pegar os instrumentos para iniciar o procedimento, como se sente?", contendo cinco alternativas de resposta, sendo que o número de cada uma delas constitui seu próprio peso. Após respondidas as questões, o operador deve realizar a soma das respostas e classificar a ansiedade, de acordo com uma tabela em: muito pouco ansioso (até 5 pontos), levemente ansioso (de 6 a 10 pontos), moderadamente ansioso (de 11 a 15 pontos) e extremamente ansioso (de 16 a 20 pontos). Para a análise de associação, as respostas de cada pergunta foram dicotomizadas pela mediana em baixa ansiedade e alta ansiedade.

O OHIP foi elaborado por Slade e Spencer ${ }^{12,25}$ e originalmente, era composto por 49 itens (OHIP-49). Posteriormente, foi desenvolvida sua forma simplificada, constituída por 14 itens, sob a denominação de Oral Health Impact Profile-short form (OHIP-14). O OHIP-14 é composto por 14 questões, dispostas em sete domínios (limitação funcional, dor física, desconforto psicológico, incapacidade física, incapacidade psicológica, incapacidade social e desvantagem social). As respostas de cada pergunta do OHIP14 foram pontuadas com os seguintes valores: 0 = nunca, $1=$ raramente, $2=$ às vezes, $3=$ com frequência, 4 = sempre, com pontuação máxima possível chegando a 56 pontos e dicotomizadas e definidas como presença de impacto, com valor 1, as respostas "com frequência" e "sempre"; e sem impacto, com valor 0 , as respostas "às vezes", "raramente" e "nunca".

\section{Análise estatística}

Os dados coletados foram analisados pelo software SPSS (Statistical Package for Social Sciences, IBM Inc., USA) versão 22.0. Inicialmente, foi realizada análise descritiva para caracterização da amostra. Em seguida, foi realizado o teste qui-quadrado de Pearson, para verificar a associação entre as variáveis categóricas, com o nível de significância de 95\% ( $<<0,05)$.

\section{Considerações éticas}

Os preceitos de pesquisa com seres humanos da Declaração de Helsinque e da Resolução 466/12 do Conselho Nacional de Saúde foram seguidos. O estudo foi realizado mediante aprovacão do Comitê de Ética em Pesquisa (CEP) da UFVJM, além da autorização prévia do Departamento de Odontologia da UFVJM, para acesso aos prontuários e uso das informações para análise e divulgação científica. 


\section{Resultados}

Foram localizados 280 prontuários de pacientes atendidos no SUO da UFVJM, correspondentes ao período de um ano de atendimento. Foram excluidos da amostra 40 prontuários, por não preencherem os critérios de inclusão.

Dos 240 prontuários analisados, verificou-se que $63,3 \%(n=152)$ dos pacientes eram do sexo feminino. A idade variou de 18 a 66 anos, com média de 33,2 anos, sendo a faixa etária de maior frequência aquela composta por pacientes de 18 a 35 anos $(60,8 \% \mathrm{n}=146)$, enquando a de 36 a 66 anos correspondeu a $39,2 \%(\mathrm{n}=94)$ da amostra.

Sobre a frequência da dor pré-operatória nos pacientes, segundo a escala HP-VAS, 13,3\% ( $\mathrm{n}=$ 32) não apresentaram dor; $5,4 \%(n=13)$ apresentaram dor tênue; $9,2 \%(\mathrm{n}=22)$, dor fraca; $7,5 \%(\mathrm{n}=18)$, dor suave; $20,8 \%(\mathrm{n}=50)$, dor moderada; $18,8 \%(\mathrm{n}=45)$, dor forte; $11,3 \%(\mathrm{n}$ $=27)$, dor intensa e 6,3\% $(n=15)$, dor máxima.

A frequência de ansiedade pré-operatória nos pacientes, segundo a escala CDAS, classificou $33,3 \%(\mathrm{n}=80)$ dos pacientes como levemente ansiosos; $29,6 \%(\mathrm{n}=71)$, como muito pouco ansiosos; $29,6 \%(n=71)$, como moderaramente ansiosos e 7,5\% ( $\mathrm{n}=18)$, como extremamente ansiosos.

Sobre a frequência do impacto na qualidade de vida por domínio, $66,7 \%(\mathrm{n}=160)$ dos pacientes reportaram dor física; $57,9 \%(\mathrm{n}=139)$, desconforto psicológico; 44,2\% $(\mathrm{n}=106)$, incapacidade física; $39,2 \%(\mathrm{n}=94)$, incapacidade psicológica; $28,8 \%(\mathrm{n}=69)$, incapacidade social, $23,3 \%(\mathrm{n}=56)$ limitação social e 14,6\% ( $\mathrm{n}=35)$ apresentaram limitação funcional.

Relação estatisticamente significativa foi observada entre dor versus idade e dor e sexo (Tabela 1). A Tabela 2 reporta associação estatisticamente significativa entre dor e qualidade de vida, nos domínios desconforto psicológico $(\mathrm{p}=<0,001)$, incapacidade física $(\mathrm{p}=<0,001)$, incapacidade psicológica ( $\mathrm{p}=<0,001)$, incapacidade social ( $\mathrm{p}$ $=<0,001)$ e desvantagem social $(\mathrm{p}=0,006)$.

Pela análise entre dor e ansiedade, as quatro perguntas da Corah's Detal Anxiety Scale não apresentaram resultados estatisticamente significativos (Tabela 3 ).

Na Tabela 4 observa-se associação positiva entre ansiedade e qualidade de vida, com resultado estatisticamente significativo nos dominios desconforto psicológico $(\mathrm{p}=0,009)$, incapacidade física $(\mathrm{p}=0,016)$, incapacidade psicológica ( $\mathrm{p}$ $=0,011)$ e desvantagem social $(\mathrm{p}=0,028)$.

\section{Discussão}

O uso de indicadores sociodentais, baseados na autopercepção e nos impactos odontológicos, oferece vantagens importantes para o planejamento e a provisão dos serviços odontológicos, destacando a mudança na ênfase dos aspectos puramente biológicos para os psicológicos e sociais $^{21}$, buscando assim entender os anseios, os medos e os desejos do paciente, para que o atendimento odontológico ocorra de forma individualizada e integral, promovendo, desse modo, a saúde e o bem-estar do paciente. A aplicação de indicadores subjetivos na área de saúde bucal trouxe uma grande contribuição para os estudos epidemiológicos na Odontologia ${ }^{26}$. Este novo enfoque tem sido muito útil para subsidiar o planejamento em saúde. O diferencial deste estudo consiste no uso de indicadores subjetivos em populações atendidas nos Serviços de UO, que, até o presente momento, foi pouco documentado na literatura científica.

Os pacientes atendidos no SUO da UFVJM, foram, em sua maioria, do sexo feminino. A mesma tendência foi observada em outros estudos que avaliaram o perfil de pacientes atendidos nos $\mathrm{SUO}^{1,2,27-29}$. Os autores sugerem que a maior prevalência de mulheres pode ocorrer devido a

Tabela 1. Distribuição de frequência da relação entre dor versus idade e dor e sexo dos pacientes atendidos no Serviço de Urgência Odontológica. Diamantina: 2014-2015.

\begin{tabular}{|c|c|c|c|c|}
\hline & Presença de dor $\mathbf{n}(\%)$ & Ausência de dor n(\%) & p-valor ${ }^{*}$ & IC 95\% \\
\hline \multicolumn{5}{|l|}{ Idade } \\
\hline 18-35 & $54(39,7)$ & $82(60,3)$ & 0,843 & $0,945(0,543-1,645)$ \\
\hline $36-65$ & $33(38,4)$ & $53(61,6)$ & & \\
\hline \multicolumn{5}{|l|}{ Gênero } \\
\hline Feminino & $54(37,8)$ & $89(62,2)$ & 0,558 & $1,182(0,675-2,071)$ \\
\hline Masculino & $33(41,8)$ & $46(58,2)$ & & \\
\hline
\end{tabular}

`Teste Qui-Quadrado de Pearson. 
Tabela 2. Distribuição da relação entre Dor versus impacto na Qualidade de Vida por domínio do OHIP-14.

\begin{tabular}{|c|c|c|c|c|c|}
\hline & & $\begin{array}{c}\text { Presença de dor } \\
n^{\mathbf{o}}(\%)\end{array}$ & $\begin{array}{c}\text { Ausência de dor } \\
n^{\circ}(\%)\end{array}$ & $\mathbf{P}^{\star}$ & IC 95\% \\
\hline \multicolumn{6}{|l|}{ Domínio 1} \\
\hline \multirow[t]{2}{*}{ Limitação funcional } & Sem impacto & $71(37,2)$ & $120(62,8)$ & 0,127 & $1,803(0,841-3,867)$ \\
\hline & Com impacto & $16(51,6)$ & $15(48,4)$ & & \\
\hline \multicolumn{6}{|l|}{ Domínio 2} \\
\hline \multirow[t]{2}{*}{ Dor física } & Sem impacto & $71(37,2)$ & $120(62,8)$ & 0,127 & $1,803(0,841-3,867)$ \\
\hline & Com impacto & $16(51,6)$ & $15(48,4)$ & & \\
\hline \multicolumn{6}{|l|}{ Domínio 3} \\
\hline \multirow[t]{2}{*}{ Desconforto psicológico } & Sem impacto & $24(25,3)$ & $71(74,7)$ & $<0,001$ & $2,912(1,632-5,196)$ \\
\hline & Com impacto & $63(49,6)$ & $64(50,4)$ & & \\
\hline \multicolumn{6}{|l|}{ Domínio 4} \\
\hline \multirow[t]{2}{*}{ Incapacidade física } & Sem impacto & $32(25,3)$ & $93(74,4)$ & $<0,001$ & $3,899(2,205-6,894)$ \\
\hline & Com impacto & $55(57,3)$ & $41(42,7)$ & & \\
\hline \multicolumn{6}{|l|}{ Domínio 5} \\
\hline \multirow{2}{*}{ Incapacidade psicológica } & Sem impacto & $41(29,9)$ & $96(70,1)$ & $<0,001$ & $2,762(1,575-4,844)$ \\
\hline & Com impacto & $46(54,1)$ & $39(45,9)$ & & \\
\hline \multicolumn{6}{|l|}{ Domínio 6} \\
\hline \multirow[t]{2}{*}{ Incapacidade social } & Sem impacto & $50(31,1)$ & $111(68,9)$ & $<0,001$ & $3,423(1,855-6,315)$ \\
\hline & Com impacto & $37(60,7)$ & $24(39,3)$ & & \\
\hline \multicolumn{6}{|l|}{ Domínio 7} \\
\hline \multirow[t]{2}{*}{ Desvantagem social } & Sem impacto & $59(34,3)$ & $113(65,7)$ & 0,006 & $2,438(1,284-4,628)$ \\
\hline & Com impacto & $28(56,0)$ & $22(44,0)$ & & \\
\hline
\end{tabular}

${ }^{*}$ Teste Qui-Quadrado de Pearson.

Tabela 3. Distribuição da relação entre dor versus ansiedade por pergunta do CDAS e somatória da ansiedade.

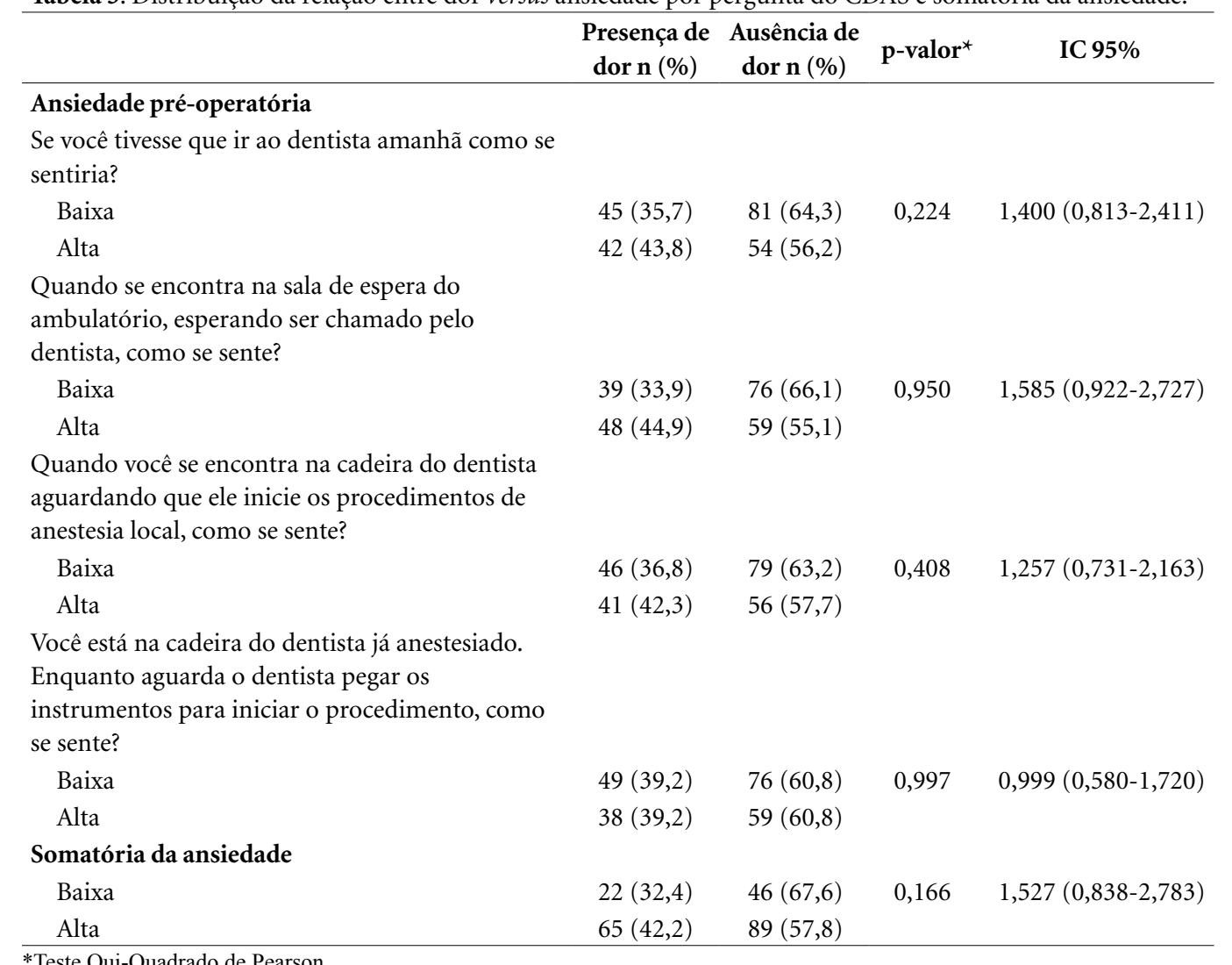

*Teste Qui-Quadrado de Pearson. 
Tabela 4. Distribuição da relação entre ansiedade versus impacto na qualidade de qualidade de vida por domínio do OHIP-14.

\begin{tabular}{|c|c|c|c|c|}
\hline & $\begin{array}{c}\text { Presença de } \\
\text { Ansiedade n(\%) }\end{array}$ & $\begin{array}{c}\text { Ausência de } \\
\text { Ansiedade n(\%) }\end{array}$ & $\mathbf{p}^{\star}$-valor & IC 95\% \\
\hline \multicolumn{5}{|l|}{ Qualidade de vida } \\
\hline \multicolumn{5}{|c|}{ Domínio 1} \\
\hline \multicolumn{5}{|c|}{ Limitação funcional } \\
\hline Sem impacto & $141(68,8)$ & $64(31,2)$ & 0,179 & $1,816(0,754-4,374)$ \\
\hline Com impacto & $28(80,0)$ & $7(20,0)$ & & \\
\hline \multicolumn{5}{|l|}{ Domínio 2} \\
\hline \multicolumn{5}{|l|}{ Dor física } \\
\hline Sem impacto & $52(65,0)$ & $28(35,0)$ & 0,194 & $1,465(0,823-2,610)$ \\
\hline Com impacto & $117(73,1)$ & $43(26,9)$ & & \\
\hline \multicolumn{5}{|c|}{ Domínio 3} \\
\hline \multicolumn{5}{|c|}{ Desconforto psicológico } \\
\hline Sem impacto & $62(61,4)$ & $39(38,6)$ & 0,009 & $2,103(1,198-3,691)$ \\
\hline Com impacto & $107(77,0)$ & $32(23,0)$ & & \\
\hline \multicolumn{5}{|c|}{ Domínio 4} \\
\hline \multicolumn{5}{|c|}{ Incapacidade física } \\
\hline Sem impacto & $85(63,9)$ & $48(36,1)$ & 0,016 & $2,038(1,139-3,646)$ \\
\hline Com impacto & $83(78,3)$ & $23(21,7)$ & & \\
\hline \multicolumn{5}{|c|}{ Domínio 5} \\
\hline \multicolumn{5}{|c|}{ Incapacidade psicológica } \\
\hline Sem impacto & $94(64,4)$ & $52(35,6)$ & 0,011 & $2,184(1,190-4,006)$ \\
\hline Com impacto & $75(79,8)$ & $19(20,2)$ & & \\
\hline \multicolumn{5}{|c|}{ Domínio 6} \\
\hline \multicolumn{5}{|c|}{ Incapacidade social } \\
\hline Sem impacto & $115(67,3)$ & $56(32,7)$ & 0,091 & $1,753(0,910-3,375)$ \\
\hline Com impacto & $54(78,3)$ & $15(21,7)$ & & \\
\hline \multicolumn{5}{|c|}{ Domínio 7} \\
\hline \multicolumn{5}{|c|}{ Desvantagem social } \\
\hline Sem impacto & $123(66,8)$ & $61(33,2)$ & 0,028 & $2,281(1,078-4,827)$ \\
\hline Com impacto & $46(82,1)$ & $10(17,9)$ & & \\
\hline
\end{tabular}

*Teste Qui-Quadrado de Pearson.

uma maior preocupação com a saúde e a estética nesse grupo ${ }^{27}$. Outra possível explicação para esta maior demanda do sexo feminino é justificada por fatores culturais, pois as mulheres são mais predispostas a buscarem atendimentos clínicos, o que pode ser observado também em outros estudos $^{27-29}$.

A faixa etária de 18 a 35 anos foi a que mais utilizou o SUO. Com o avançar da faixa etária, houve um decréscimo contínuo no número de usuários do SUO. Este decréscimo da procura com o avançar da idade pode ser devido ao grande contingente populacional acima de 51 anos ser formado por pessoas parcial ou totalmente desdentadas. Com isso, apresentam uma menor chance de procurar o SUO devido a problemas odontológicos $^{1,30}$.
Como a dor de origem dental possui alta prevalência, esta apresenta-se como um problema de saúde pública, causando grande impacto negativo na qualidade de vida das pessoas. Em função disto, a atenção às urgências odontológicas possui especial importância à saúde da população ${ }^{31}$. No presente estudo, a maioria dos pacientes apresentou dor de intensidade moderada à severa, revelando que a procura ao SUO só ocorreu quando o problema começa a se agravar. Resultado similar foi encontrado em um estudo transversal no serviço de SUO de Goiás, no qual $50,4 \%$ dos pacientes apresentavam dor de intensidade moderada à severa ${ }^{32}$.

A ansiedade é um importante obstáculo na procura de cuidados a saúde ${ }^{33}$, tendo consequências prejudiciais, representando um sério desafio 
epidemiológico para os profissionais que cuidam da saúde bucal ${ }^{34,35}$. A prevalência de ansiedade relacionada a fatores odontológicos foi estimada, no mundo, em $9 \%{ }^{36}$. Estudos realizados em países de primeiro mundo, como Suécia ${ }^{37}$, EUA $^{38}$ e Dinamarca ${ }^{39}$, demonstraram prevalência de ansiedade em $6,7 \%, 10 \%$ e $10,2 \%$ da população, respectivamente. No presente estudo, foi constatado que $70,4 \%$ da amostra manifestou ansiedade, de um modo geral, independentemente da sua severidade (leve, moderada ou extrema). Um dado preocupante foi um total de 7,5\% da amostra ser classificada como extremamente ansiosa. Apesar da relativa baixa prevalência, estes pacientes demandaram maior cuidado durante a abordagem e adequado preparo do operador. Embora não tenha sido objeto deste estudo caracterizar a ansiedade e a sua etiologia, houve evidência de ansiedade e necessidade de uma abordagem diferenciada, para obter maior cooperação durante o atendimento clínico. Estudo realizado na Faculdade de Odontologia de Araraquara mostrou alta prevalência de ansiedade ao tratamento odontológico (95\%), sendo que $16,7 \%$ dos pacientes foram classificados como extremamente ansiosos ${ }^{40}$, com resultados superiores ao presente estudo. Estes fatos evidenciam que a grande maioria dos pacientes atendidos nos SUOs apresenta-se ansiosa, estes devem ser identificados, incentivados, acalmados e cuidadosamente monitorados. Alguns pacientes diagnosticados como ansiosos necessitam de pré-medicação ansiolítica leve durante o decorrer do tratamento odontológico, a fim de melhorar seu comportamento, reduzindo seus níveis de ansiedade ${ }^{41}$ e também otimizando o próprio tratamento.

Estudos apontam que a ansiedade pode modular o impacto na qualidade de vida ${ }^{2}$. Um estudo realizado no Reino Unido com 3.000 indivíduos evidenciou que pacientes que se mostraram ansiosos durante o tratamento odontológico relataram o dobro do impacto negativo em sua qualidade de vida ${ }^{17}$. Resultado similar foi encontrado em um estudo transversal realizado na Índia, com amostra de 1.235 indivíduos, avaliando a associação entre ansiedade ao tratamento odontológico e qualidade de vida ${ }^{18}$. Na visão dos autores, este resultado se justifica porque ansiedade e impacto decorreram de características psicológicas, envolvendo aspectos de afetividade negativa. Outra possível explicação seria uma maior negligência dos indivíduos com sua condição bucal, o que geraria maiores volume e complexidade de tratamentos, implicando no aumento da ansiedade, na diminuição da procura, na piora da condição bucal e, consequentemente, em maior impacto negativo na qualidade de vida ${ }^{17,18}$.

O OHIP-14 foi utilizado neste estudo por ser uma ferramenta sensível para se avaliar o impacto da saúde bucal na qualidade de vida em adultos $^{42}$, sendo validado em vários idiomas, como chinês $^{43}$, senegalês ${ }^{44}$, hebraico ${ }^{45}$, sueco ${ }^{46}$, italiano $^{47}$, alemão ${ }^{48}$, grego ${ }^{49}$ e português ${ }^{50,51}$.

Estudo com amostra de 2027 adultos canadenses $^{52}$ observou que a dor física e o desconforto psicológico foram as dimensões que apresentaram maior impacto sobre a qualidade de vida dos indivíduos. Outros estudos também detectaram resultados semelhantes ${ }^{19,53}$. Estas pesquisas sustentam a hipótese de que os pacientes que buscaram atendimento no SUO apresentaram fortes dores na boca, acarretando estresse, preocupação, nervosismo e incômodo ao comer algum alimento ${ }^{53}$. Contudo, no presente estudo, dor física e limitação funcional foram os dois únicos domínios que não estiveram associados estatisticamente com pior qualidade de vida. Este resultado discorda dos reportados por Locker \& Quiñonez $^{52}$ e sugere que uma nova abordagem deva ser realizada para a elucidação do mesmo e a aplicação de outros testes, dentre eles, o de percepção da condição de saúde bucal.

Houve associação estatisticamente significativa entre dor e qualidade de vida em cinco dos sete domínios do OHIP-14, corroborando com um estudo similar em que a presença da dor impactou negativamente na qualidade de vida ${ }^{16}$.

Não foi observada associação entre dor, idade e sexo, diferindo dos achados literários, que relataram maior prevalência de dor entre as mulheres, devido a aspectos fisiológicos, perceptivos, sociais, comportamentais e, em especial, fatores hormonais ${ }^{54}$.

Na amostra estudada, não foi possível estabelecer relação entre dor e ansiedade pré-operatória. Em busca nas bases de dados, não foram localizados outros estudos que abordassem dor, ansiedade e qualidade de vida dos pacientes atendidos nos SUOs, o que dificulta a comparação dos resultados com a literatura. Foi observada associação entre ansiedade e qualidade de vida, com resultados estatisticamente significativos em quatro domínios do OHIP. Um estudo com amostra de 3500 adultos suecos evidenciou que indivíduos com alta ansiedade apresentam maior impacto negativo na qualidade de $v i d a^{55}$, corroborando os resultados do presente estudo. A constatação de que maior ansiedade foi associada ao impacto negativo na qualidade de vida já era esperada e está bem documentada ${ }^{56-60}$. Também 
foi verificada associação entre a alta ansiedade e consequências funcionais e psicossociais na qualidade de vida ${ }^{55}$. A razão para isto pode ser devido à negligência dos indivíduos ansiosos em relação à sua saúde oral, de tal forma que apresentam altos níveis de doença não tratada, repercutindo negativamente na qualidade de vida ${ }^{17}$. Esforços devem ser realizados para se detectar pacientes ansiosos e, assim, melhorar o impacto na qualidade de vida dos mesmos ${ }^{17,18}$.

Uma limitação do estudo foi a ausência de dados a respeito da condição socioeconômica dos pacientes atendidos no SUO, que não constavam no prontuário clínico.

Apesar de assumir os limites de interferência e da associação, por se tratar de estudo transversal, com utilização de uma amostra que abrangeu apenas parte da população assistida pelo SUO, o presente estudo fornece dados importantes para o planejamento e a organização dos serviços de atendimentos odontológicos, principalmente de urgência, considerando, além das necessidades clínicas, os fatores psicológicos relacionados com o estado de saúde bucal.

\section{Conclusão}

A dor e a ansiedade exercem significativo impacto negativo na qualidade de vida dos pacientes atendidos em SUO causando desconforto psicológico, incapacidades física, psicológica e social. Esse estudo é relevante para o planejamento de programas e ações voltadas para a saúde bucal dos pacientes atendidos nos SUO, priorizando aqueles com maiores impactos psicossociais produzidos pelos problemas bucais.

\section{Colaboradores}

FD Verli, SMC Santos, JA Soares foram responsáveis pela concepção e delineamento, MF Queiroz foi responsável pela revisão da literatura, coleta dos dados, redação da versão inicial do manuscrito e redação da versão final do manuscrito. PCP Paiva contribuiu na análise estatística, interpretação dos dados e na revisão crítica do manuscrito. SA Marinho participou da revisão crítica e versão final do manuscrito. A versão final foi aprovada por todos os autores. 


\section{Referências}

1. Pinto EC, Barros VJA, Coelho MQ, Costa SM. Urgências odontológicas em uma Unidade de Saúde vinculada à Estratégia Saúde da Família de Montes Claros, Minas Gerais. Arq Odontol 2013; 48(3):166-174.

2. Kanegane K, Penha SS, Borsatti MA, Rocha RG. Ansiedade ao tratamento odontológico em atendimento de urgência. Rev Saude Publica 2003; 37(6):786-792.

3. Flumignan JDP, Sampaio Neto LFD. Atendimento odontológico em unidades de emergência: caracterização da demanda. Rev Bras Odontol 2014; 71(2):124129.

4. Farmakis ETR, Palamidakis FD, Skondra FG, Nikoloudaki G, Pantazis N. Emergency care provided in a Greek dental school and analysis of the patients' demographic characteristics: a prospective study. Int Dent $J$ 2016; 66(5):280-286.

5. Currie CC, Stone SJ, Durham J. Pain and problems: a prospective cross-sectional study of the impact of dental emergencies. J Oral Rehabi 2015; 42(12):883-889.

6. Shqair AQ, Gomes GB, Oliveira A, Goettems ML, Romano AR, Schardozim LR, Bonow ML, Torriani DD. Dental emergencies in a university pediatric dentistry clinic: a retrospective study. Braz Oral Res 2012; 26(1):50-56

7. Sischo L, Broder HL. Oral Health-related Quality of Life What, Why, How, and Future Implications. J Dent Res 2011; 90(11):1264-1270.

8. Slade GD. Oral health-related quality of life is important for patients, but what about populations?. Community Dent Oral Epidemiol 2012; 40(s2):39-43.

9. Zucoloto ML, Maroco J, Campos JADB. Impact of oral health on health-related quality of life: a cross-sectional study. BMC Oral Health 2016; 16(1):55.

10. Mendonça BMC, Cimões R, Araújo ACS, Caldas AFJ, Silva PV. Impacto do número de dentes presentes no desempenho de atividade diárias: estudo piloto. Cien Saude Colet 2010; 15(3):775-784.

11. Guerra MJC, Greco RM, Leite ICG, Ferreira EF, Paula MVQD. Impact of oral health conditions on the quality of life of workers. Cien Saude Colet 2014; 19(12):477786.

12. Slade GD. Derivation and validation of a short-form oral health impact profile. Community Dent Oral Epidemiol 1997; 25(4):284-290.

13. Liu P, Mcgrath C, Cheung GSP. Improvement in Oral Health-related Quality of Life after Endodontic Treatment: A Prospective Longitudinal Study. J Endod 2014; 40(6):805-810.

14. Rigo L, Basso K, Pauli J, Cericato GO, Paranhos LR, Garbin RR. Satisfaction with life, dental experience and self-perception of oral health among the elderly. Cien Saude Colet 2015; 20(12):3681-3688

15. Papaioannou W, Oulis CJ, Yfantopoulos J. The oral health related quality of life in different groups of senior citizens as measured by the OHIP-14 questionnaire. Oral Biol Dent 2015; 3(1):1.

16. Carvalho FS. Medo, ansiedade e dor de dente em adolescentes: impacto na qualidade de vida, na saúde bucal e no acesso aos serviços de saúde [tese]. São Paulo: Faculdade de Odontologia de Bauru; 2012.

17. Mcgrath C, Bedi R. The association between dental anxiety and oral health-related quality of life in Britain. Community Dent Oral Epidemiol 2004; 32(1):67-72.
18. Kumar S, Bhargav P, Patel A, Bhati M, Balasubramanyam G, Duraiswamy P, Kulkarni S. Does dental anxiety influence oral health-related quality of life? Observations from a cross-sectional study among adults in Udaipur district, India. J Oral Sci 2009; 51(2):245-254.

19. Gomes AS, Abegg C. The impact of oral health on daily performance of municipal waste disposal workers in Porto Alegre, Rio Grande do Sul State, Brazil. Cad Saude Publica 2007; 23(7):1707-1714.

20. Prado RL, Saliba NA, Carbim CA, Moimaz SA. Oral impacts on the daily performance of Brazilians assessed using a sociodental approach: analyses of national data. Braz Oral Res 2015; 29(1):1-9.

21. Bennadi D, Reddy CVK. Oral health related quality of life. J Int Soc Prev Community Dent 2013; 3(1):1-6.

22. Heft MW, Parker SR. An experimental basis for revising the graphic rating scale for pain. Pain 1984; 19(2):153161.

23. Corah NL. Development of a dental anxiety scale. $J$ Dent Res 1969; 48(4):596.

24. Hu LW, Gorenstein C, Fuentes D. Portuguese version of Corah's Dental Anxiety Scale: transcultural adaptation and reliability analysis. Depress Anxiety 2007; 24(7):467-471.

25. Slade GD, Spencer AJ. Development and evaluation of the oral health impact profile. Community Dent Health 1994; 11(1):3-11.

26. Miotto MHMB, Barcellos LA, Velten DB. Avaliação do impacto na qualidade de vida causado por problemas bucais na população adulta e idosa em município da Região Sudeste. Cien Saude Colet 2012; 17(2):397-406.

27. Paula JS, Oliveira M, Soares MR, Chaves MCA, Mialhe FL. Epidemiological profile of patients treated in the emergency clinic of the School of Dentistry at the Federal University of Juiz de Fora. Arq Odontol 2012; 48(4):257-262.

28. Lee S, Borsatti MA, Penha SS, Buscaríolo IA, Domaneschi C, Tortamano IP. Aspectos epidemiológicos e sociodemográficos do Setor de Urgência da Faculdade de Odontologia da Universidade de São Paulo. Rev Pos Grad 2007; 13(4):299-306.

29. Silva CHV, Araújo ACS, Fernandes RSM, Alves KA, Pelinca RN, Dias YC. Perfil do serviço de pronto atendimento odontológico da Universidade Federal de Pernambuco Odontologia. Clin Cientif 2009; 8(3):229235.

30. Brasil. Ministério da Saúde (MS). Projeto SB Brasil 2010. Pesquisa Nacional de Saúde Bucal: Resultados Principais. Brasília: MS; 2011.

31. Rios LE. Atenção às urgências odontológicas em unidades de pronto-atendimento do sistema único de saúde. Goiânia: Universidade Federal de Goiás; 2013.

32. Murrer RD, Francisco SS, Endo, MM. Ansiedade e medo no atendimento odontológico de urgência. Rev Odont Bras Central 2015; 23(67):196-201.

33. Shapiro M, Melmed RN, Sgan-Cohen HD, Eli I, Parush S. Behavioural and physiological effect of dental environment sensory adaptation on children's dental anxiety. Eur J Oral Sci 2007; 115(6):479-483. 
34. Lahmann C, Schoern R, Henningsen P, Ronel J, Muehlbacher M, Loew T, Tritt K, Nickel M, Doering S. Brief relaxation versus music distraction in the treatment of dental anxiety: a randomized controlled clinical trial. $J$ Am Dent Associ 2008; 139(3):317-324.

35. Morse Z, Takau AF. Dental anxiety in Fiji. Pac Health Dialog 2004; 11(1):22-25.

36. Klingberg G, Broberg, AG. Dental fear/anxiety and dental behaviour management problems in children and adolescents: a review of prevalence and concomitant psychological factors. Int J Paediatr Dent 2007; 17(6):391-406.

37. Hakeberg M, Berggren U, Carlsson SG. Prevalence of dental anxiety in an adult population in a major urban area in Sweden. Community Dent Oral Epidemiol 1992; 20(2):97-101.

38. Ronis DL. Updating a measure of dental anxiety: reliability, validity, and norms. J Dent Hyg 1994; 68(5):228233

39. Singh KA, Moraes ABA, Bovi Ambrosano GM. Medo, ansiedade e controle relacionados ao tratamento odontológico. Pesq Odont Bras 2000; 14(2):131-136.

40. Chaves AM, Loffredo LCM, Valsecki A, Chavez OM, Campos JAD. Estudo epidemiológico da ansiedade dos pacientes ao tratamento odontológico. Rev Odontol UNESP 2006; 35(4):263-268.

41. Ferraz GR, Nascimento TMT, Menezes Fo PF, Fernandes RSM. Avaliação do grau de ansiedade dos pacientes no pré-cirúrgico de Implantodontia. Full Dent Sci 2014; 5(18):258-262.

42. Tsakos G, Allen PF, Steele JG, Locker D. Interpreting oral health-related quality of life data. Community Dent Oral Epidemiol 2012; 40(3):193-200.

43. Wong MC, Lo E, Mcmillan AS. Validation of a Chinese version of the Oral Health Impact Profile (OHIP). Community Dent Oral Epidemiol 2002; 30(6):423-430.

44. Ekanayake L, Perera I. Validation of a Sinhalese translation of the Oral Health Impact Profile-14 for use with older adults. Gerodontology 2003; 20(2):95-99.

45. Kushnir D, Zusman S, Robinson PG. Validation of a Hebrew version of the Oral Health Impact Profile 14. $J$ Public Health Dent 2004; 64(2):71-75.

46. Hägglin C, Berggren U, Hakeberg M, Edvardsson A, Eriksson M. Evaluation of a Swedish version of the OHIP-14 among patients in general and specialist dental care. Swed Dent J 2007; 31(2):91-101.

47. Segù $M$, Collesano V, Lobbia $S$, Rezzani C. Cross-cultural validation of a short form of the Oral Health Impact Profile for temporomandibular disorders. Community Dent Oral Epidemiol 2005; 33(2):125-130.

48. Hassel AJ, Steuker B, Rolko C, Keller L, Rammelsberg P, Nitschke I. Oral health-related quality of life of elderly Germans-comparison of GOHAI and OHIP-14. Community Dent Health 2010; 27(7):242-247.
49. Papagiannopoulou V, Oulis CJ, Papaioannou W, Antonogeorgos G, Yfantopoulos, J. Validation of a Greek version of the oral health impact profile (OHIP-14) for use among adults. Health Qual Life Outcomes 2012; 10:7.

50. Ferreira CA, Loureiro CA, Araújo VE. Psycometrics properties of subjetive indicator in children. Rev Saude Publica 2004; 38(3):445-452.

51. Zucoloto ML, Maroco J, Campos JA. Psychometric Properties of the Oral Health Imapct Profile and New Methodological Approach. J Dent Res 2014; 93(7):645650.

52. Locker D, Quiñonez C. To what extend do oral disorders compromise the quality of life? Community Dent Oral Epidemiol 2011; 39(1):3-11.

53. Batista MJ, Perianes LB, Hilgert JB, Hugo FN Sousa Mda. The impacts of oral health on quality of life in working adults. Braz Oral Res 2014; 28(1):1-6.

54. Lira MOSC, Carvalho MFAA. Dor aguda e relação de gênero: diferentes percepções em homens e mulheres. Rev Rene 2013; 14(1):71-81.

55. Carlsson V, Hakeberg M, Wide Boman U. Associations between dental anxiety, sense of coherence, oral health-related quality of life and health behaviour - a national Swedish cross-sectional survey. BMC Oral Health 2015; 15:100.

56. Johansson V, Axtelius B, Söderfeldt B, Sampogna F, Paulander J, Sondell K. Multivariate analyses of patient financial systems and oral health-related quality of life. Community Dent Oral Epidemiol 2010; 38(5):436-444.

57. Ng SK, Leung WK. A community study on the relationship of dental anxiety with oral health status and oral health-related quality of life. Community Dent Oral Epidemiol 2008; 36(4):347-356.

58. Vermaire JH, De Jongh A, Aartman IH. Dental anxiety and quality of life: the effect of dental treatment. Community Dent Oral Epidemiol 2008; 36(5):409-416.

59. Crofts-Barnes NP, Brough E, Wilson KE, Beddis AJ, Girdler NM. Anxiety and quality of life in phobic dental patients. J Dent Res 2010; 89(3):302-306.

60. Gisler V, Bassetti R, Mericske-Stern R, Bayer S, Enkling $\mathrm{N}$. A cross-sectional analysis of the prevalence of dental anxiety and its relation to the oral health-related quality of life in patients with dental treatment needs at a university clinic in Switzerland. Gerodontology 2012; 29(2):e290-e296.

Artigo apresentado em 18/11/2016

Aprovado em 27/06/2017

Versão final apresentada em 29/06/2017 